\title{
Mathematics of predictive food microbiology
}

\author{
József Baranyi *, Terry A. Roberts \\ Institute of Food Research, Reading Laboratory, Reading, RG6 2EF, UK \\ Received 30 May 1994; accepted 7 August 1994
}

\begin{abstract}
Commonly encountered problems related to modelling bacterial growth in food are analysed from a mathematical point of view. Modelling techniques and terms, some misused, are discussed and an attempt is made to clarify how, and under what conditions, they may be used. A theoretical framework is given to provide a basis in which mathematical models having been used in predictive microbiology can be embedded. By using several simplifying idealizations as a compromise between the complexity of the biological system and the available data, a practically usable model becomes available.
\end{abstract}

Keywords: Mathematical modelling; Predictive food microbiology; Bacterial growth; Differential equation; Cell kinetics

\section{Introduction}

The traditional approach to establishing food safety is via challenge tests using the pathogens of concern. That approach has long been regarded as expensive, slow, demanding on facilities and microbiological skills, and more recently as providing only modest assurance that a product formulation will be safe in the food chain. Moreover, knowledge acquired is not cumulative, and when a product formulation, or the temperature-time profile to which it is subjected changes, challenge tests must be repeated. An alternative is to understand more fully the responses of the microbes of concern to the key controlling factors in the food environment, to build a cumulative store of knowledge, and to develop the means to interpolate calculated microbial responses. The long term prospect of not

\footnotetext{
" Corresponding author.
} 
having to repeat challenge tests when the responses are confidently assured was embodied in the term 'predictive microbiology'.

Since its inception, predictive microbiology has prompted many animated discussions and been subject to certain criticism (see, for example, Hedges, 1991). When a new concept is born, it is not unusual for papers to be published containing controversial terminology and describing mere curve fitting procedures as mathematical models. Admittedly, there is no mechanistic background behind many, so-called, 'predictive models', but, as Box and Draper (1987) mentioned, empirical models can develop to become more and more mechanistic as the information on the system being modelled increases. Therefore, we feel, this 'naive' stage has been a useful, and perhaps even a necessary, beginning. Some criticism could, however, have been avoided if researchers had been more careful when using mathematical terms and tools and had tried to integrate the concept of predictive microbiology into related scientific disciplines more exactly.

In this paper, some misused terms and curve fitting techniques are discussed first, then a mathematical framework is shown in which predictive food microbiology can be embedded. We use some notations frequently applied in mathematial textbooks. A vector will be denoted by bold italics: $x=\left(x_{1} \ldots x_{n}\right)$ means that the vector $x$ has $n$ entries, $x_{1}, x_{2}, \ldots ., x_{n}$. These entries can be time- $(t-)$ dependent variables, too, in which case $x(t)=\left(x_{1}(t) \ldots x_{n}(t)\right)$ is a time-dependent vector variable. The notation $\boldsymbol{y}=\boldsymbol{f}(\boldsymbol{x})$ means that there is a functional relationship, denoted by $f$, between the components of the two vectors: each component of $y$ can be expressed as a function of the components of $\boldsymbol{x}$.

\section{Misused terms and techniques}

\subsection{Model and function}

The term 'model' has been defined in several excellent reviews on the mathematical modelling of microbiological processes (see, for example, Roels and Kossen, 1978). A purely empirical model, like the quadratic response surface for the environment dependence of a parameter of a bacterial population (as in Gibson et al., 1988) is a model, too, but its aim is nothing more than a smooth representation of the experimental results. It is a (regression) model in the sense used in the regression analysis of statistics where the aim is the numerical representation of certain responses by means of simple functions, like polynomials, without mechanistic explanation. The term mathematical model is more rigorous and refers to a set of basic hypotheses on the studied processes, some of which are possibly expressed by means of functions and (differential) equations. Therefore, from a mechanistic point of view, function and model are not equivalent terms. Function is a mathematical abstraction making it easier to describe a particular model. 
For example, the definition of the Gompertz model of growth (Turner et al., $1976)$ is based upon the hypothesis that $\mu(t)$, the instantaneous specific growth rate of a quantity (we return to its definition later again) is related to the instantaneous amount, $x(t)$, of that quantity by

$$
\mu(t)=c \cdot \ln \frac{x_{\max }}{x(t)}
$$

where $x_{\text {rnax }}$ is the maximum value of $x(t)$ and $c$ is a model constant. This leads to a differential equation, the solution of which is termed the Gompertz function. This function, completed by an additive term, was fitted by Gibson et al. (1988) to the logarithm of cell concentrations. It is appropriate to call the resulting sigmoid curve a Gompertz function (to whatever it is fitted) but, in a mechanistic sense, it obviously should not be called as Gompertz model. Nevertheless, it is true that in textbooks of regression analysis, the term 'model' is widely used for a function of a certain form, irrespective of how that function was originally derived.

\subsection{Rate and specific rate}

Let $x(t)$ be a time-dependent variable describing the variation of a certain substance, like biomass or cell concentration. The instantaneous rate of the process is the derivative of $x(t): d x(t) / d t$. The specific rate, $\mu(t)$, is defined as

$$
\mu(t)=\frac{\frac{\mathrm{d} x(t)}{\mathrm{d} t}}{x(t)}
$$

If $x(t)$ denotes the cell concentration in a bacterial culture then the growth rate is meant as the absolute increase in cell concentration per unit time, while the specific growth rate is the increase in cell concentration per unit time per cell. A simple calculation shows that if $x(t)$ is positive then

$$
\mu(t)=\frac{\mathrm{d}(\ln x(t))}{\mathrm{d} t}
$$

Therefore, the specific growth rate can be measured as the slope of the growth curve where the natural logarithm of $x(t)$ is plotted against time. If $\log _{10}$ is used instead of the natural logarithm then, the measured slope will be $\ln (10) \approx 2.3$ times less than the specific growth rate.

\subsection{Doubling time and generation time}

At a fixed time, $t_{\mathrm{fix}}$, let the value of $\mu(t)$ be $\mu\left(t_{\mathrm{fix}}\right)=\mu_{\mathrm{fix}}$. The relation for the doubling time, $T_{d}=\ln 2 / \mu_{\text {fix }} \approx 0.69 / \mu_{\text {fix }}$, means that if $\mu(t)$ remained the same then, at the time $t_{\text {fix }}+T_{d}$, the cell concentration would be double of that what it was at $t_{\mathrm{fix}}$. It is important to note that, in asynchronous cultures, $T_{d}$ is not equivalent to the mean generation time. In fact, the mean generation time can be 
estimated by $1 / \mu_{\text {fix }}$ rather than by $0.69 / \mu_{\text {fix }}$, if the cells' division can be approximated by a random birth process, such as the Poisson process (Rubinow, 1984).

\subsection{Intrinsic and controlling parameters}

Suppose that a bacterial process is modelled by an $x(t)=\mathrm{f}(p ; t)$ function, where the vector $p$, involves the model parameters. These paramcters depend on the particular bacteria used and on the (possibly time-dependent) conditions of the experiment which are influenced by the experimenter himself. It is advantageous if $p$ can be divided into $p_{1}$ and $p_{2}$ where $p_{1}$ is independent of the experimenter and $p_{2}$ depends only on him/her. In this case, we can call the components of $p_{1}$ the intrinsic parameters, and those of $p_{2}$ the controlling parameters. This is frequently the situation when the model is described by a system of differential equations, the parameters of which are intrinsic parameters, but the initial values, which must be provided to obtain a particular solution for the differential equation, are controlling parameters depending mainly on the experimenter. For example, a simple, 'lag-less' model of a batch culture is

$$
\begin{aligned}
& \frac{\mathrm{d} x(t)}{\mathrm{d} t}=\mu_{0} x(t) \\
& x(0)=x_{0}
\end{aligned}
$$

where $x(t)$ is the (time-dependent) bacterial concentration, $x_{0}$ is its value at the time $t=0$ and $\mu_{0}$ is the (constant) specific growth rate. The solution of the above, so-called, 'initial value problem' is

$$
x(t)=x_{0} e^{\mu_{0} t}
$$

Therefore, in this model, the parameter vector is $p=\left(\mu_{0}, x_{0}\right)$, where $\mu_{0}$ is an intrinsic, and $x_{0}$ is a controlling parameter. It is quite easy to destroy this clear separation by reparameterizing the model function, for example:

$$
x(t)=\left(q_{1}+q_{2}\right) e^{\left(q_{1}-q_{2}\right) t}
$$

where

$$
\begin{aligned}
& q_{1}=\frac{x_{0}+\mu_{0}}{2} \\
& q_{2}=\frac{x_{0}-\mu_{0}}{2}
\end{aligned}
$$

In this form of the model function, $q_{1}$ and $q_{2}$ are obviously not purely controlling or intrinsic parameters. As a consequence, it would not be fruitful to model $q_{1}$ or $q_{2}$ as a function of the effect of the environment on bacterial growth because both also depend on the experimenter. That modelling procedure is correct only under the assumption that $x_{0}$ is fixed and the model is used for prediction only at this fixed $x_{0}$. Such a model would have limited practical use. Nevertheless, there are cases when similar, temporary, reparameterization is useful for other reasons, like 
numerical stability or close-to-linear behaviour (Ratkowsky, 1983). It is important, however, to bear in mind that the different reparameterizations have computational, and not mechanistic, reasons.

The reparameterization of the Gompertz function was analysed by Garthright (1991) and Baranyi (1991a,b). A commonly used form of the Gompertz function is

$$
g(t)=a+c e^{-e^{-b(t-m)}}
$$

where $a, b, c, d$ are function parameters (Gibson et al., 1988; Buchanan and Phillips, 1990). From these parameters, the maximum slope of the curve is:

$$
\mu_{\max }=\frac{b c}{e}
$$

If $g(t)$ is fitted to the natural logarithm of the cell concentration, this new parameter, $\mu_{\max }$, will be an estimation of the maximum specific growth rate of that culture. Accepting that a given environment unambiguously defines the maximum specific growth rate at which a homogeneous population is able to grow (Rubinow, $1984), \mu_{\max }$ is an intrinsic parameter of the model.

The difference between the upper asymptote and the inoculum level, estimatable by $c$, clearly depends on the experimenter who can decide a higher or lower inoculum level. Hence it is not an intrinsic parameter, but nor is it purely a controlling parameter, because it obviously depends on some bacterial characteristics, toc. Therefore, the parameter

$$
b=\mu_{\max } \frac{e}{c}
$$

is not an intrinsic parameter either, because it must depend on the inoculum value. The consequence is that the environment-dependence of the parameter $b$ should not be modelled except when the inoculum level is the same, fixed, constant for both the experiments and the growth curves for which the prediction is given.

However, the above form of $g(t)$ can be useful from computational point of view, and, depending on the dataset to which it is applied, may show slightly better statistical features (as explained in Baranyi 1992a; 1992b) than the reparameterization suggested by Zwietering et al. (1991) and Garthright (1991). There is no contradiction in using the $(a, c, b, m)$-form of $g(t)$ for curve fitting and then to model the effect of the environment on the derived parameter, $\mu_{\max }=b c / e$. Note, however, that in our opinion, the Gompertz function should not be used to fit the logarithm of the bacterial concentration in any form (Baranyi et al., 1993a).

Accordingly, it is unfortunate that the parameter $b$ has been called the 'relative growth rate', as in Gibson et al. (1988) and Buchanan et al. (1990).

\subsection{The better the fit the better the model?}

Fitting a quadratic response surface to estimates of the maximum specific growth rate, or one of its transformations (e.g. log- or square-root transformation; see McMeekin et al., 1993) is a straightforward, simple solution to represent the 
Table 1

Example data for maximum specific growth rates at different $\mathrm{pH}$ values

\begin{tabular}{ll}
\hline $\mathrm{pH}$ & $\mu_{\max }$ \\
\hline 4.5 & 0.112 \\
4.5 & 0.129 \\
4.6 & 0.132 \\
4.6 & 0.123 \\
4.7 & 0.212 \\
5.0 & 0.387 \\
5.0 & 0.317 \\
5.0 & 0.378 \\
5.1 & 0.373 \\
5.5 & 0.451 \\
5.6 & 0.368 \\
5.6 & 0.459 \\
5.6 & 0.416 \\
6.0 & 0.423 \\
6.0 & 0.482 \\
6.0 & 0.469 \\
6.9 & 0.488 \\
7.0 & 0.462 \\
7.0 & 0.531 \\
7.0 & 0.506 \\
7.0 & 0.468 \\
7.0 & 0.476
\end{tabular}

environment-dependence of the modelled variable. Multivariate quadratic functions are natural generalizations of second order polynomials, or parabolas. Buchanan and Phillips (1990), judged by an $F$-test, suggested that the multivariate generalization of a third order polynomial provided a better description of their data measuring the environment-dependence of the kinetic parameters of Listeria monocytogenes. The idea was taken up by Hudson (1993), who concluded that the third order surface was a better model for his data than the quadratic surface. Before being tempted to use the multidimensional versions of the fourth, or even higher, order polynomials, it is worth investigating if this 'upgrading-approach' results in real progress.

It is well-known that, generally, the more parameters a model contains the better fit it can produce. The $R^{2}$ statistic, in itself, obviously cannot decide whether or not a new model is an improvement. Even the $F$-test, which takes the number of model parameters into account, is not an absolute measuring tool.

As an example, consider Table 1 which contains the maximum specific growth rates of an organism at various $\mathrm{pH}$ values from $\mathrm{pH}_{\min }=4.5$ to $\mathrm{pH}_{\max }=7$. Apart from the $\mathrm{pH}$, other conditions (temperature, $a_{w}$, inoculum size, etc.) were made identical for the batch cultures from which the $\mu_{\max }$ values were derived. Some curves were replicated and, due to natural variance, more $\mu_{\max }$ values belong to certain $\mathrm{pH}$ levels. The identity of the organism is not required to demonstrate our statistical point. A second and a third order polynomial were fitted to these data 
Table 2

Results of fitting a second (y1) and a third (y2) order polinomial to the data of Table 1

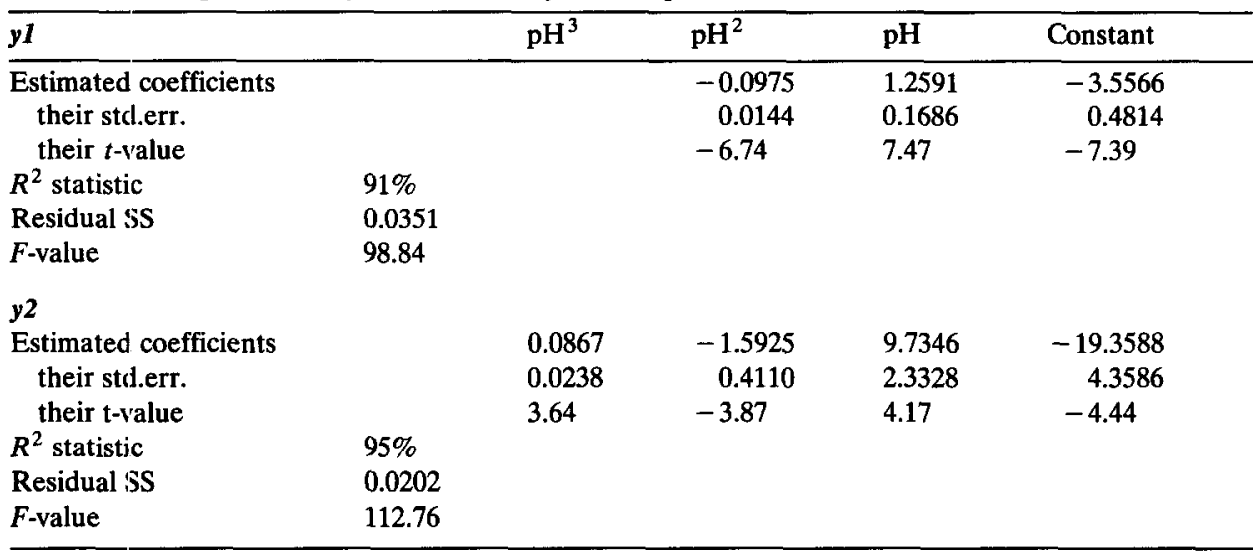

using the commercial Microsoft Excel package. The results are detailed in Table 2 and represented in Fig. 1. In a statistical sense, the third order polynomial, $y 2$, is undoubtedly better than the parabola, $y 1$. But is this 'upgrading' an appropriate way to seek for improvement? The parabola, $y 1$, suggests that, in the interval $6<\mathrm{pH}<7$, where there are no data, $\mu_{\max }$ first increases then decreases, having a

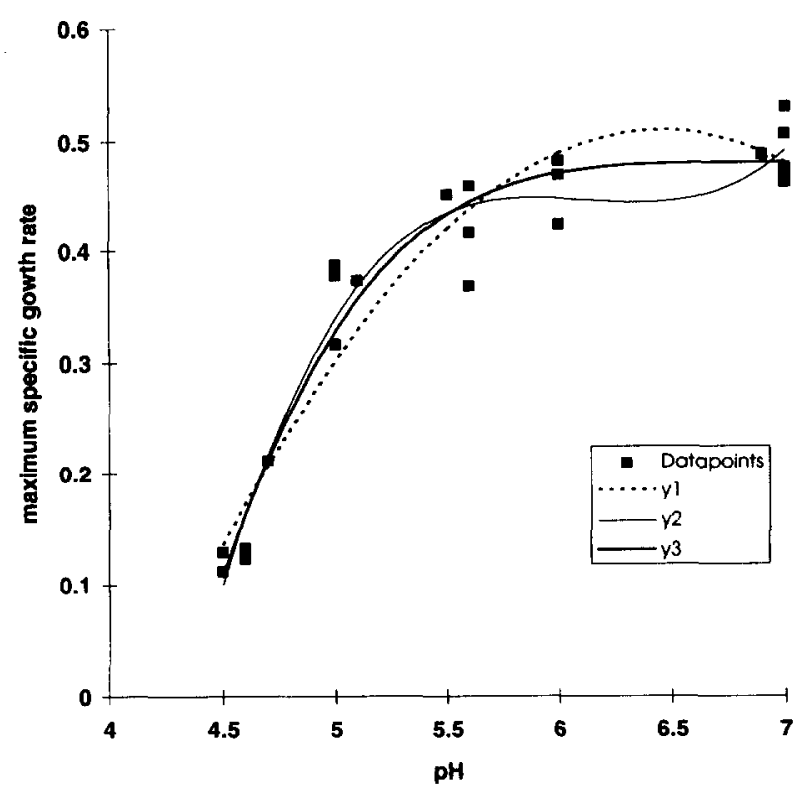

Fig. 1. Maximum specific growth rates at different $\mathrm{pH}$ values (see Table 1) fitted by a quadratic function $\left(y_{1}\right)$, a third order polynomial $\left(y_{2}\right)$ and a quadratic function of a transformation of $\mathrm{pH}: \mathrm{pH}^{\prime}=(7-\mathrm{pH})^{2}$. 
maximum at about $\mathrm{pH}=6.5$. In the same $\mathrm{pH}$ interval, the third order polynomial, $y 2$, suggests the opposite, $\mu_{\max }$ first slightly decreasing then increasing, having a local minimum at about $\mathrm{pH}=6.5$. The truth is, however, that there is no reason to suppose any local maximum or minimum below $\mathrm{pH}=7$ and, in fact, the data did not indicate it. Using either $y 1$ or $y 2$ for predictions around $\mathrm{pH}=6.5$ would probably give worse results than a simple estimation by eye. But, if we rescale the explanatory variable by introducing $\mathrm{pH}^{\prime}=(7-\mathrm{pH})^{2}$, knowing that the optimum $\mathrm{pH}$ is around 7.0, then a parabolic regression on the $\mathrm{pH}^{\prime}$ variable results in a curve along which $\mu_{\max }$ increases with $\mathrm{pH}$ in the whole experimental region ( $y 3$ of Fig. 1). This last curve is the closest of the three to that expected from experience.

This example shows that, although the response surfaces are useful from quantitative points of view, any conclusion about the shape of the function ('qualitative feature') representing the true model should be accepted with serious reservations, especially in regions where there are relatively few data like between pH 6 and 7 in the example above.

It is generally true that the higher the order of a polynomial, the more 'hills and valleys' it can produce. A higher order polynomial, as a model function, is able to follow the random, up and down, errors of the measured values. However, our aim should be to eliminate these random errors rather then to fit them. On the other hand, the degree of the polynomial used in the model determines certain 'qualitative features' of the curve. A first order polynomial does not have minimum or maximum, a second order polynomial is symmetrical around its maximum or minimum, the third order polynomial has an inflexion point, as in the example above, etc.

A possible improvement of the model is to fix some expected properties of the response function. For example, expected location of extreme points, monotonity, convexity, etc., all belong to these qualitative features. In the case above, it was a reasonable expectation that the model function should have only one extreme point, which is a maximum, and it is monotone increasing before the maximum and monotone decreasing after it. This could be achieved by keeping the second order polynomial as a model function but rescaling the explanatory variable.

A famous example for rescaling a model variable is that of Arrhenius, where $T^{\prime}=1 /\left(T-T_{0}\right)$ was introduced for the temperature $T$ with $T_{0}=-273^{\circ} \mathrm{C}$. Gibson et al. (1995) rescaled the $a_{w}$ variable, measuring the water activity, by the substitution $b_{w}=\sqrt{\left(1-a_{w}\right)}$. These examples show that, instead of higher and higher order polynomials producing more and more 'hills and valleys', it is generally more advantageous to find appropriate transformations for either the explanatory or the dependent variables. The better fit does not necessarily mean a better model, especially in terms of 'qualitative features', which play a crucial role at the later stage of using the model for prediction.

\subsection{Indirect measures of growth responses}

It is reasonable to consider the concentration of viable cells, $x(t)$, to be the basic variable in a homogeneous bacterial culture from which the instantaneous 
specific growth rate, $\mu(\mathrm{t})$, can be calculated as the derivative of $\ln x(t)$. For obvious technical reasons (automation, etc.), indirect measures of both $x(t)$ and $\mu(\mathrm{t})$ are frequently reported in the literature. It is crucial to establish the relationship between the desired quantity and what, in fact, is measured. The role of these, so-called, calibration functions is essentially equivalent to that of the rescaling transformations mentioned in Section 2.5 above.

Turbidity and conductance measurements claim to be approximately linearly proportional to $x(t)$ (Meynell and Meynell, 1965; Jason, 1983). This means that, if the measured quantity is $q$, then $q=\mathrm{f}(x)$, where $f$ is a linear calibration function: $f(x)=a \cdot x+b$. If $b$ is different from 0 then neither $q$ nor $\log q$ is linearly proportional to $\log x$. Hence, in a strict sense, the rate of change in $q$ should not be used to estimate the viable count specific growth rate unless the proportionality of $q$ (turbidity, conductance, etc.) to the original cell concentration, $x$, has been established over the complete matrix of environmental variables (temperature, $\mathrm{pH}$, $\left.a_{w}\right)$. Nor should the viable count models describing $x(t)$ be directly applied to model $q(t)$. New calibration function, or other considerations, should be taken into account to model $q(t)$ and/or to compare it with the viable count model.

\section{A mathematical model for bacterial growth}

A mathematical model is a set of, possibly not explicitly stated, assumptions, some of which are formulated by (differential) equations. In biology, the real system is extremely complex, so its model must inevitably include simplifying idealizations. These occur, for example, when one variable measures a feature which is, in fact, a composite of several contributing features, or when a variable is considered to be constant in time and/or homogeneous in space although it is known to be time-dependent and heterogeneous. 'The question is, however, not whether we introduce simplifying idealizations but where these idealizations should be made and how extensive they should be.' (Gold, 1977).

Frederickson et al. (1967) described some fundametal properties which any reasonable mathematical model of microbial kinetics should have. The models used in various disciplines, like biotechnology and food microbiology, should differ only in the aspect where the above mentioned idealizations and simplifications are placed and how extensive they are. Baranyi and Roberts (1994) listed several reasons why food microbiology should not simply copy the mathematical models used in biotechnology. Below we follow those idealizing and simplifying steps which make a model practically usable in food microbiology.

The basic hypothesis is that the cell kinetics is determined by three classes of variables:

- Intracellular conditions defining the instantaneous state of a cell. We assume that this state can be measured by the concentrations of certain biochemical entities, like biomass, enzymes or RNA, DNA. The per cell concentrations of 
these $n$ entities will be denoted by $z(\mathrm{t})=\left(z_{1}(\mathrm{t}), \ldots, z_{n}(t)\right)$. We also refer to $z(\mathrm{t})$ as internal or physiological state vector.

- Extracellular conditions that are changed by the bacterial metabolism. These quantities are involved in a vector, $c(t)$, called the 'external state vector of category 1 '.

- Extracellular conditions that are independent of the growing culture. The vector variable involving these quantities is termed the 'external state vector of category 2 ' and is denoted by $D(t)$. As it is indicated in the notation, it possibly changes with time. The most commonly modelled entry in $D(t)$ is usually the temperature, $T(t)$.

The same classification of the variables affecting cell growth can be found also in, for example, Hills and Wright (1994). In Baranyi et al. (1993a), the composition of the external state vectors formed the environment: $E(t)=(c(t), D(t))$ where the $E(t)$ function has a discontinuity at inoculation.

To realize a practically usable model, several simplifying hypotheses are made.

(1) We assume that the bacterial population is homogeneous, i.e. we disregard the possible space distribution of the cells. We do not take into account the natural biological variance, i.e. we disregard that $z(t)$ has also probability distribution. Disregarding these distributions is equivalent to considering the entries of the physiological state vector as averages in the physical and probability space. The error due to this assumption will be called the homogeneity error.

(2) The mathematical form of our basic hypothesis is that the kinetics of the system can be expressed as

$$
\begin{aligned}
& \frac{\mathrm{d} z(t)}{\mathrm{d} t}=f(z(t), c(t) ; D(t)) \\
& \frac{\mathrm{d} c(t)}{\mathrm{d} t}=g(z(t), c(t) ; D(t))
\end{aligned}
$$

The semicolon, separating the variable $D(t)$ from $z(t)$ and $c(t)$, indicates that the functions $f$ and $g$ depend on the actual state of the growth-independent external conditions, $D(t)$. The simplest example is when the value of the rate constants in $f$ and $g$ depend on the external temperature.

If $D(t)$ is constant then the system above is called autonomous (Frederickson, 1967). This means that the 'future' of the $c, z$ variables (their values after $t_{0}$ ) depends only on their values at $t_{0}$ and does not depend on their 'past' (values before $t_{0}$ ). In other words, an injured cell, having fully recovered, generates the same successive subpopulations as if it had been healthy throughout its history. We do not consider the possible error (do the cells have memory of prior injury?) of this autonomy assumption.

Note that, if the solution of (2) is substituted into (1), then the obtained differential equation for $z(t)$ is non-autonomous. This was emphasised by Baranyi et al. (1993a). However, under reasonable assumptions, its solution converges to a delayed solution of its autonomous counterpart; the rate of this convergence was estimated by Baranyi et al. (1993b). 
We consider the cell concentration of the homogeneous population, $x(t)$ the principal quantity to be modelled. A binary fission takes place if the internal and external conditions allow it, therefore the instanteneous specific growth rate, $\mu(t)$, which can be considered as an instantaneous measure of the birth rate per cell per unit time, depends on $z(t)$ and $c(t)$ :

$$
\mu(t)=\phi(z(t), c(t) ; D(t))
$$

After these assumptions, the cell concentration can be calculated from the first order differential equation

$$
\frac{\mathrm{d} x(t)}{\mathrm{d} t}=\mu(t) x(t)
$$

Apart from certain singular situations, the system of first order differential Eqs. (1)-(4) has a unique solution, if the initial conditions

$$
z\left(t_{0}\right)=z_{0} ; \quad c\left(t_{0}\right)=c_{0} ; \quad x\left(t_{0}\right)=x_{0}
$$

and the external $D(t)$ function are provided. In other words, the variation of the system with time, after $t_{0}$, is determined by the $f, g, \phi$ model functions and

- the value of the internal state vector, $z(t)$, at $t_{0}$;

- the value of the category 1 external state vector, $c(t)$, at $t_{0}$;

- the category 2 external state vector, $D(t)$, as a function of time.

Note that $x(t)$ could always be included in $c(t)$ as a variable characterizing the 'crowdedness' around a single cell. Because of its distinguished role, however, $x(t)$ and its differential equation were singled out from the components of $c(t)$ and (2).

(3) Another simplification is the assumption that $z(t), c(t)$ and $D(t)$ can be represented by just a few components, i.e. it is sufficient to characterize the internal/external environment by some main factors only. When restricted to a few environmental factors, we introduce a certain error. This will be referred to as completeness error in what follows.

Since growing cells produce heat, in a strict sense, the temperature is not a category 2 external variable and should be a component of $c(t)$ and not $D(t)$. However, the small contribution of that heat is neglected. This example illustrates that the border between the category 1 and category 2 external variables can, to a certain extent, be arbitrary, taking practical considerations into account. An external factor of category 2 can become that of category 1 as the information on the system increases and the particular model develops further. It is also possible that different, extra- and intracellular, concentrations of the same substance, like water, are treated as different entries and the connection between them are described by certain (for example, diffusion-) equations built in the functions $f$ and $g$ of the Eqs. (1) and (2).

The homogeneity error can be decreased by making the variables of the system random or space-dependent (heterogeneous systems). The completeness error can be decreased by taking more and more variables into account when creating $z(t)$, $c(t)$ and $D(t)$. Both improvements are rather labour-intensive when trying to identify the model parameters from measured data. What is more, the error of 


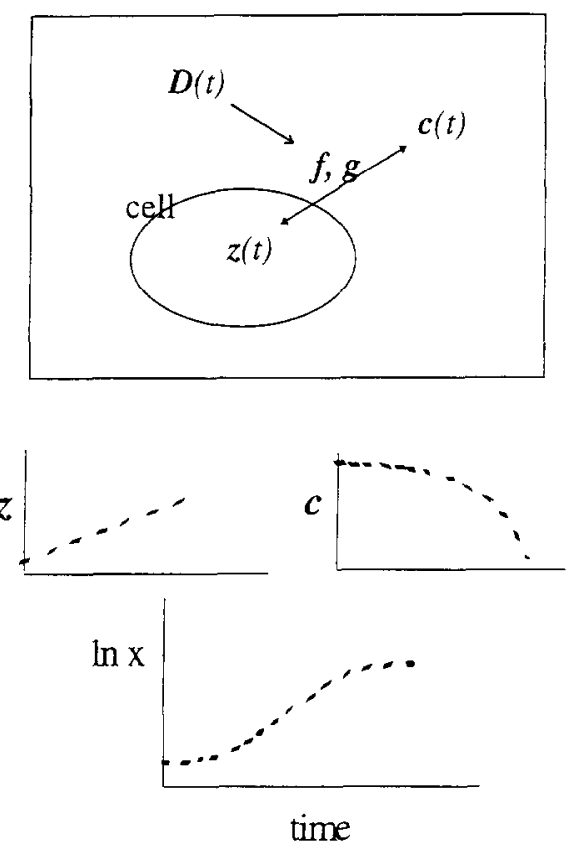

Fig. 2. The intra- and extracellular conditions of a cell, $z(t)$ and $c(t)$, mutually affect each other. Their rate is described by the functions $f(\mathbf{z}, \mathbf{c})$ and $g(\mathbf{z}, \mathbf{c})$. The parameters in $f$ and $g$ depend on the actual state of those external conditions, involved in $D(t)$, which are not affected by the bacterial metabolism. During a typical (sigmoid shaped) bacterial growth curve, $z(t)$ improves during the lag phase and $c(t)$ deteriorates in the stationary phase.

prediction may even increase if the collected data are not sufficient in quantity or accuracy. An exaggeratedly detailed model may even become practically useless due to the lack of good quality data. It is frequently more efficient to improve the 'qualitative' features (shape, analytical characteristics) of the function in the Eqs. (1)-(4). The error due to modelling the 'true' kinetics by the model functions $f, g$ and $\phi$ will be referred to as model function error.

Note that, as the term 'model' is not used in a statistical sense (see point 1 in the Introduction), different model functions are really different functions here. In statistics, different model functions may represent the same function with different parameterization (Ratkowsky, 1983).

The mathematical construction above is demonstrated in Fig. 2. The kinetics between $z(t)$ and $c(t)$ are described by the functions $f$ and $g$. These functions, however, depend on the actual state of the external conditions, like temperature, atmosphere, etc., which are not affected by the growth. The probability of a binary fission in the small $[t, t+\Delta t]$ interval is $\mu(\mathrm{t}) \Delta \mathrm{t}$, where $\mu(\mathrm{t})=\phi(z, c ; D(t))$. Assuming that the cell division happens randomly, for example, according to a Poisson process, the change of the population concentration with time can be described by Eq. (4). 
As an illustration, we show how to embed different models in the structure above.

\subsection{Monod's model}

In this model (see, for example, Frederickson et al. 1967), $z(t)$ and $c(t)$ have only one entry: $z_{1}(t)$ measures the mass of a cell and $c_{1}(t)$ is the substrate concentration around the cell.

The equations, corresponding to (1) and (2) are:

$$
\begin{aligned}
& \frac{\mathrm{d} z_{1}(t)}{\mathrm{d} t}=k_{1} \frac{c_{1}(t)}{K_{c}+c_{1}(t)} z_{1}(t) \\
& \frac{\mathrm{d} c_{1}(t)}{\mathrm{d} t}=-\frac{1}{Y} \frac{\mathrm{d} z_{1}(t)}{\mathrm{d} t}
\end{aligned}
$$

where $k_{1}, K_{c}$ and $Y$ are model constants, depending on the external conditions, $D(t)$ only. Assuming that binary fission takes place when the mass of a cell reaches a critical (constant) value, the specific growth rate of the total mass is equal to the specific growth rate of the cell concentration, thus providing an equation corresponding to (3):

$$
\mu(t)=\frac{\mathrm{d} z_{1}(t)}{\mathrm{d} t}
$$

To decicle whether the parameters $k_{1}, K_{c}$ and $Y$ depend on the exteral conditions, $D(t)$, and how, is a basic task in biotechnology. For the rate $k_{1}$, for example, the most common model is that of Arrhenius inasmuch as $D(t)$ is primarily defined by the temperature.

\subsection{The model of Hills and Wright (1994)}

To describe the lag phase, in addition to the growth rate, a posible idea is to introduce more compartments in $z(t)$. In the model of Hills and Wright (1994), the internal state vector has two components, $z_{1}(t)$, and $z_{2}(t)$ representing the so-called excess biomass and the total biomass of a cell, respectively. $z_{1}(t)$ is the difference between the actual biomass of the cell $\left(z_{2}(t)\right)$ and the minimum mass below which a cell is no longer viable. This minimum mass, denoted by $s_{\min }$, is assumed to be constant. Because the cell number is proportional to the total biomass, the system of equations corresponding to (1) reads:

$$
\begin{aligned}
& \frac{\mathrm{d} z_{1}(t)}{\mathrm{d} t}=\left(k_{1}-k_{2} z_{1}(t)\right) z_{2}(t) \\
& z_{2}(t)=z_{1}(t)+s_{\min }
\end{aligned}
$$

where $k_{1}$ and $k_{2}$ depend on the external environment, $c(t)$ and $D(t)$, according to versions of Monod's and Arrhenius' function. The system was solved explicitly only 
in the case when the external conditions were constant, therefore no upper asymptote was obtained with the explicit solution. Unlike in Monod's model, for (3), the equation

$$
\mu(t)=\frac{z_{1}(t)}{s_{\min }}
$$

was applied. Solving the equations in constant external conditions, it was deduced that

$$
\mu(t)=k_{1} \alpha(t)=k_{1} \frac{k_{2} e^{k_{1} t}-k_{2} e^{-k_{2} t}}{k_{2} e^{k_{1} t}+k_{1} e^{-k_{2} t}}
$$

where the constants depend on the external conditions. Since the value of $\alpha(\mathrm{t})$ is between 0 and 1 , and as $t$ increases $\alpha(t)$ converges to 1 monotone increasingly, it describes the process of adjustment in the lag phase, where the maximum specific growth rate, $k_{1}$ is gradually approached. The rate of that convergence is defined by $k_{2}$, similarly to the model below.

\subsection{The model of Baranyi and Roberts (1994)}

The internal state vector, $z(t)$, has two components where $z_{1}(t)$ represents the per cell quantity of a critical substance causing the bottleneck in the growth (such as RNA or ATP) and $z_{2}(t)$ is the biomass of a cell. The category 1 external state vector, $c(t)$, has only one component, the nutrient concentration. It is assumed that the lack of the critical substance has a limiting effect according to the Michaelis-Menten kinetics (limitation 'from the left'). In a similar manner, decreasing nutrient concentration causes limitation in growth 'from the right':

$$
\begin{aligned}
\frac{\mathrm{d} z_{1}(t)}{\mathrm{d} t} & =k_{1} z_{1}(t) \\
\frac{\mathrm{d} z_{2}(t)}{\mathrm{d} t} & =\frac{z_{1}(t)}{K_{z}+z_{1}(t)} k_{2} \frac{c_{1}(t)}{K_{c}+c_{1}(t)} z_{2}(t) \\
\frac{\mathrm{d} c_{1}(t)}{\mathrm{d} t} & =-\frac{1}{Y} \frac{\mathrm{d} z_{2}(t)}{\mathrm{d} t}
\end{aligned}
$$

where $k_{1}, k_{2}, Y, K_{z}$ and $K_{c}$ are model paramenters, depending only on the category 2 external variables. The cell concentation, $x(t)$ is assumed to be proportional to the total biomass, therefore

$$
\mu(t)=\frac{\frac{\mathrm{d} z_{2}(t)}{\mathrm{d} t}}{z_{2}(t)}=\frac{z_{1}(t)}{K_{z}+z_{1}(t)} k_{2} \frac{c_{1}(t)}{K_{c}+c_{1}(t)}
$$

Note that the first equation above already contains a simplification because $z_{1}(t)$ initially increases, but then decreases as the cell divides (see more details in 
Baranyi et al., 1995). The separation of the effects of the internal and external environment is reflected by the above formula very clearly. The specific growth rate depends on the physiological state of the cell (via $z_{1}$ ); on the category 1 external conditions (via $c_{2}$ ); and on the category 2 external conditions (via the model constants).

Denote $\nu=k_{1}, \mu_{\max }=k_{2}$ and

$$
\begin{aligned}
& \alpha(t)=\frac{z_{1}(t)}{K_{z}+z_{1}(t)} \\
& u(t)=\frac{c_{1}(t)}{K_{c}+c_{1}(t)}
\end{aligned}
$$

The values of the functions $\alpha(t)$ and $u(t)$ are between 0 and 1 . This is why $k_{2}$ can be called the maximum specific growth rate. The function $\alpha(t)$ describes the process of adjustment controlled by the accumulation of the critical substance, $z_{1}(t)$. Therefore, $\alpha(\mathrm{t})$ converges to 1 , as $t$ increases, similarly to the model of Hills and Wright (1994). The limiting function $u(t)$ describes the transition to the stationary phase.

For practical purposes, some simplifications were devised in accord with biological considerations.

The nutrient limitation, in food, generally appears at high cell concentration only. Considering the nutrient infinite, however, results in limitless growth. For the sake of simplicity and because, in food microbiology, modelling the effect of nutrient limitation is less significant, $u(t)$ is approximated by a simple limiting function depending on a maximum cell density parameter:

$$
u(t) \approx 1-\left(\frac{x(t)}{x_{\max }}\right)^{m}
$$

where $x_{\text {rnax }}$ is the maximum cell concentration, $m$ is a curvature parameter characterizing the transition of the growth curve to the stationary phase. This is a technical realization of a previous note that $x(t)$ can always be involved into the components of $c(t)$. The model obtained for $x(t)$ is:

$$
\frac{\mathrm{d} x(t)}{\mathrm{d} t}=\frac{z_{1}(t)}{K_{z}+z_{1}(t)} \mu_{\max }\left(1-\left(\frac{x(t)}{x_{\max }}\right)^{m}\right) x(t)
$$

where

$$
\frac{\mathrm{d} z_{1}(t)}{\mathrm{d} t}=\nu z_{1}(t)
$$

That is, the effect of the bacterial growth on the extracellular environment is expressed as a self-limiting growth where the stationary phase is caused by the high cell concentration itself. The error due to this simplification appears, obviously, only at high cell concentrations.

The advantage of this simplification is that, if $D(t)$ is constant, then the system of the above differential equations has an explicit solution, which makes the curve 
fitting procedures easier. As has been published by Baranyi and Roberts (1994), the solution for the natural logarithm of the cell concentration, $y=\ln x(t)$, is:

$$
y(t)=y_{0}+\mu_{\max } A(t)-\frac{1}{m} \ln \left(1+\frac{e^{m \mu_{\max } A(t)}-1}{e^{m\left(y_{\max }-y_{0}\right)}}\right)
$$

where $y_{0}=\ln x\left(t_{0}\right)$, the natural logarithm of the cell concentration at $t=t_{0}$; $y_{\max }=\ln x_{\max }$, the natural logarithm of the maximum cell concentration.

The function $A(t)$ plays the role of a gradual delay in time:

$$
A(t)=t+\frac{\ln \left(e^{-\mu_{\max } t}+e^{-h_{0}}-e^{-\nu t-h_{0}}\right)}{\mu_{\max }}
$$

where $h_{0}=-\ln \alpha_{0}$ and

$$
\alpha_{0}=\frac{z_{1}\left(t_{0}\right)}{K_{z}+z_{1}\left(t_{0}\right)} \text {. }
$$

The parameter $\alpha_{0}$ is called the physiological state of the cells at $t=t_{0}$.

A further simplifying assumption was that $m=1$ and $\nu=\mu_{\max }$ (for the reasoning, see Baranyi and Roberts, 1994). In addition to the several computational advantages compared with other sigmoid functions, the main use of the model is that it predicted bacterial growth response well, even if the temperature changed with time during the lag and exponential phases (Baranyi et al., 1995). It is very important to remember that, generally, the system (5), (6) does not necessarily have an explicit solution. The $\mu_{\max }(D(t))$ function must not be simply substituted into the formulae above but the differential Eqs. (5) and (6) should be solved numerically on computer.

Partly because of biological, and partly because of computational, considerations, further simplifications were devised to obtain a practically usable model matching the quality of available data:

(a) The specific growth rate of the cells adapt to the temperature changes instanteneously, i.e. in the $\mu_{\max }(D(t))$ function, there is no delay term.

(b) The $\mu_{\max }(D)$ function can be approximated by a multivariate polynomial of the components of $D$.

(c) If the cells have the same history (e.g the subculturing and inoculation procedures are carefully standardized) then the initial physiological state, $\alpha_{0}$, can be considered constant. Consequently, $h_{0}=\ln \alpha_{0}$ is also constant and can be used to characterize the initial physiological state. It is assumed that, in a certain range of the environmental factors involved in $D$, the parameter $h_{0}$, which proved to be the product of the maximum specific growth rate and the lag, is independent of the actual values of those environmental factors (see Baranyi and Roberts, 1994).

Applying these simplifications certainly increases the model function error and restricts the region where the model is valid. For example, Baranyi et al. (1995) 
remarkecl that, at low water activity, the product of the lag and the growth rate is not constant, and that cells can grow abruptly after a relatively long lag phase. However, the fewer parameters to be identified, the more acurate is the estimation they produce. Such constraints towards parsimony are vital to get smaller errors when using the model for prediction.

The idealizations and simplifying assumptions followed through above constitute a particular mathematical model applicable to bacterial growth in environments characterizing food.

\section{Discussion}

After fixing the structure of the model, the task is to find the values of the model parameters, thus defining a particular pair of $f$ and $g$ model functions. The criterion for these functions is generally the goodness of fit to measured data. As mentioned above, each step in the model construction contains a certain error. In the main, these were classified as

- homogeneity error;

- completeness error;

- model function error.

When identifying the model parameters by fitting them to measured data, two other types of errors emerge:

- measurement error;

- numerical procedure error.

In the term 'numerical procedure error', we include all those errors originating from the numerical mathematics of the model fitting procedure. Note that, as in the case of the so-called ill-conditioned problems (Stoer and Bulirsch, 1981), it is sometimes impossible to realize a usable model.

As an example of estimating the contribution of the different error categories to the overall error, consider the Brochothrix thermosphacta model of McClure et al. (1993). From fitting the growth curves individually by a sigmoid curve (first step), the standard errors of the maximum specific growth rates, $\mu_{\max }$, were estimated, on average, as $8 \%$ of the estimatcd $\mu_{\max }$ valucs. Observe that in Fig. 1, although not $B$. thermosphacta, several replicates of growth curves gave maximum specific growth rate values with about $10 \%$ deviance. This means that a response surface fitted to the $\ln \mu_{\max }$ values (second step) is expected to give about $\exp (0.08) \approx 0.1$ for the standard error of regression (also called Residual Mean Square Error, RMSE) in that paper. This is a consequence of transforming the modelled variable into its natural logarithm. Instead, the RMSE values were $\operatorname{RMSE}_{1}=0.29$ and $\mathrm{RMSE}_{2}=0.27$, depending on whether the Gompertz function or the function of Baranyi et al. (1993) was used in the first step. The meaning of the RMSE is as follows: if experimental growth curves are generated under conditions described in 
the paper, the observed $\mu_{\max }$ values will differ, on average, from the predicted $\mu_{\max }$ by about $\pm 30 \%$, because $\exp (0.27) \approx 0.30$. Because of the multiplicative nature of this error term, the same can be said for the observed minimum doubling times.

Since the laboratory experiments were carried out in media, which can be considered to be homogeneous environment, and the conditions, apart from the intentionally changed and measured environmental factors, were identical, the homogeneity error and the completeness error did not make a significant contribution to the RMSE. Nor did the numerical procedure error, as the calculated indicators showed. Therefore, the next estimation can be given: in the expcrimental region, the use of the quadratic surface, as a model for the $\mu_{\max }(D)$ function, ca. tripled the originally $10 \%$ measurement error of $\mu_{\max }$. That is, for similar experiments, the maximum specific growth rate can be predicted to about $\pm 30 \%$ accuracy, two thirds of which is the model function error. Note, however, that this is an average and, in fact, the error distribution varies in the mathematical space of the environmental variables.

The authors collected about 100 independent measures of doubling times of $B$. thermosphacta from the literature. The predicted doubling times were almost always 'fail-safe' because the laboratory medium was optimal for the bacteria apart from those effects which were modelled.

Apart from the 20\% model function error, the above model completed its task, without labour-intensive effort to reduce the homogeneity and completeness error, inasmuch as the aim was to model the greatest bacterial growth expected in a given environment.

In real food situation, improving the model would appear to be attainable by decreasing the homogeneity and completeness errors. However, when complicating the model by introducing space-dependent variables and additional environmental factors, it is important to remember the warnings outlined in Section 2.5 of the Introduction. Further parameters should be added only when they are justified, and when they are supported by data relevant to the new parameter(s) and of appropriate quality. More parameters usually mean a better fit to the measured data (i.e. the data from which the model was derived), but may mean reduced accuracy when the model is used for predictions, becausc of lack of parsimony.

A dilemma faces those attempting to model microbial growth reponses in foods. Foods are varied and usually complex structures. The microbial response is biochemically complex, and is likely to vary with the circumstances. One solution is to try to take account of all the known and anticipated properties such as cell distribution, heterogeneity of the microbial population etc. to make an all-encompassing model. This demands a very detailed understanding of those properties and how they are affected by the conditions prevailing in the food and its environment. Such detailed information may not be available, and may be difficult, or costly, to acquire. It is also possible that the intermediate stages of the complex model development will not yield a practically useful preliminary model.

An alternative approach is to make certain assumptions and simplifications, which must be biologically justifiable, and model relatively simple measures of 
microbial growth and death with respect to relatively few descriptors of the environment to determine first how much of the observed biological response can be accounted for by this simplified approach. That which cannot be explained would then require more complex efforts.

We have been gratified that the latter approach, making assumptions and simplifications, has already yielded models that give useful and usable estimates of the microbial responses in a wide range of foods and circumstances pertinent to food microbiology and the food industry.

\section{References}

Baranyi, J. (1992a) Notes on reparameterization of bacterial growth curves. Food Microbiol. 9, 169-174.

Baranyi, J. (1992b) Notes on reparameterization of bacterial growth curves II. Food Microbiol. 9, $265-267$.

Baranyi, J. and Roberts, T.A. (1994) A dynamic approach to predicting bacterial growth in food. Int. J. Food Microbiol. 23, 277-294.

Baranyi, J., Roberts, T.A. and McClure, P.J. (1993a) A non-autonomous differential equation to model bacterial growth. Food Microbiol. 10, 43-59.

Baranyi, J., Roberts, T.A. and McClure, P.J. (1993b) Some properties of a non-autonomous deterministic growth model describing the adjustment of the bacterial population to a new environment. IMA J. Math. Appl. Med. Biol. 10, 293-299.

Baranyi, J., Robinson, T.P., Shukla A. and Mackey B.M. (1994) Predicting growth of Brochothrix thermosphacta at changing temperature. Int. J. Food Microbiol. (in press).

Buchanan, R.L. and Phillips, J.G. (1990). Response surface model for predicting the effects of temperature, $\mathrm{pH}$, sodium chloride content, sodium nitrite concentration and atmosphere on the growth of Listeria monocytogenes. J. Food Protect., 53, 370-376.

Frederickson, A.G., Ramkrishna, D. and Tsuhiya, H.M. (1967) Statistics and dynamics of procaryotic cell populations. Math. Biosci. 1, 327-374.

Garthright, W.E. (1991) Refinements in the prediction of microbial growth curves. Food Microbiol. 8, 239-248.

Gibson, A.M., Bratchell, N. and Roberts, T.A. (1988) Predicting microbial growth: growth responses of salmonellae in a laboratory medium as affected by $\mathrm{pH}$, sodium chloride and storage temperature. Int. J. Food Microbiol. 6, 155-178.

Gibson, A.M., Baranyi, J., Pitt, I., Eyles M.J. and Roberts, T.A. (1994) Predicting fungal growth: the effect of water activity on four species of Aspergillus. Int. J. Food Microbiol. 23, 419-431.

Gold, H.J. (1977) Mathematical Modelling of Biological Systems. Wiley, New York.

Hedges, A. (1991) Predictive microbiology - or is it? Lett. Appl. Microbiol., 13, 217-219.

Hills, B.P. and Wright, K.M. (1994) A new model for bacterial growth in heterogeneous systems. J. Theor. Biol. 168, 31-41.

Hudson, I.A. (1993) Construction and comparison of response surface kinetic models for the Yersinia enterocolitica type strain and a food isolate under aerobic conditions. Int. J. Food Microbiol. 18, 201-2199.

Jason, A.C. (1983) A deterministic model for monophasic growth of batch cultures of bacteria. Antonie van Leeuvenhok 49, 513-536.

McClure, P.J., Baranyi, J., Boogard, E., Kelly, T.M. and Roberts, T.A. (1993) A predictive model for the combined effect of $\mathrm{pH}$, sodium chloride and storage temperature on the growth of Brochothrix thermosphacta. Int. J. Food Microbiol. 19, 161-178.

McMeekin, T.A., Olley, J.N., Ross, T. and Ratkowsky, D.A. (1993) Predictive Microbiology. John Wiley \& Sons Ltd., Chichester, UK.

Meynell, G.G., Meynell, E. (1965) Theory and Practice in Experimental Bacteriology. Cambridge University Press, Cambridge, UK. 
Ratkowsky, D.A. (1983) Nonlinear regression modeling. Marcel Dekker, New York.

Ratkowsky, D.A., Lowry, R.K., McMeekin, T.A., Stokes, A.N. and Chandler, R.E. (1983) Model for bacterial culture growth rate throughout the entire biokinetic temperature range. J. Bacteriol. 154, $1222-1226$.

Roels, J.A. and Kossen, N.W.F. (1978) On the modelling of microbial metabolism. In: M.J. Bull (editor), Progress in Industrial Microbiology, 14. Elsevier, Amsterdam.

Rubinow, S.I. (1984) Cell kinetics. In: L.A. Segel (editor), Mathematical Models in Molecular and Cell Biology. Cambridge University Press, Cambridge.

Stoer, J. and Bulirsch, R. (1981) Introduction to Numerical Analysis. Springer, Berlin.

Turner, M.E., Bradley, E.L., Kirk, K.A. and Pruitt, K.M. (1976) A theory of growth. Math. Biosci. 29, 367-373

Zwietering, M.H., Jongenburger, I., Rombouts, F.M. and van't Riet, K. (1990) Modelling of the bacterial growth curve. Appl. Environ. Microbiol. 56, 1875-1881. 